
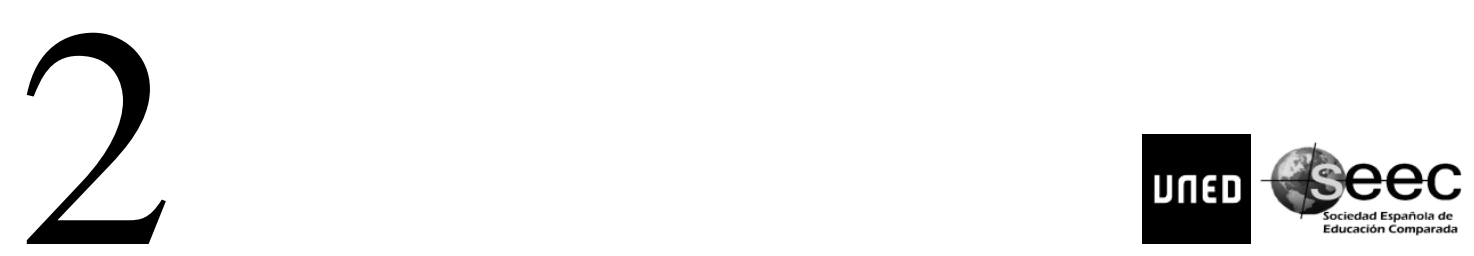

\title{
LOS CAMBIOS RECIENTES EN LA FORMACIÓN INICIAL DEL PROFESORADO EN ESPAÑA: UNA REFORMA INCOMPLETA
}

\section{Recent changes in initial teacher training in Spain: An unfinished reform}

\author{
Alejandro Tiana *
}

\section{RESUMEN}

El sistema educativo español viene asistiendo durante la última década a un proceso sostenido de cambios, que no parece haber llegado todavía a su punto final. Tras haberse completado en el año 2000 las transferencias educativas a las comunidades autónomas comenzó un proceso de transformaciones internas, tanto a escala autonómica como central, que ha afectado a muy diversos ámbitos del sistema educativo. La formación del profesorado, tanto la inicial como la permanente, también se han visto afectadas por este conjunto de cambios. En líneas generales, puede decirse que la transformación principal consistió en la sustitución del modelo establecido por la Ley General de Educación de 1970 por el actual modelo basado en el sistema de grados y postgrados del Espacio Europeo de Educación Superior (EEES). El artículo comienza abordando el contexto internacional en que esta reforma se ha gestado y desarrollado para pasar después a presentar y comentar el proceso de cambio experimentado como consecuencia de la aprobación en 2006 de la Ley Orgánica de Educación (LOE) y las características principales del nuevo modelo

\footnotetext{
* Universidad Nacional de Educación a Distancia (España).
} 
adoptado. Finalmente se argumenta por qué se puede considerar que se trata de una reforma incompleta, analizando sus principales limitaciones y las posibles vías de avance.

PALABRAS CLAVE: reformas educativas, formación inicial del profesorado, profesión docente, espacio europeo de educación superior.

\section{ABSTRACT}

The Spanish education system is experiencing in the last decade a sustained process of change which does not seem to have reached yet its final point. After having finished in 2000 the process of transfer of responsibilities in education to the so-called autonomous communities, a new process of internal changes in different fields did start both at a state and regional level. Initial and in-service teacher training have been also affected by this process. Generally speaking, the main change consisted in replacing the model set up by the 1970 Education Act by a new one based in the development of the European space for higher education. The paper starts by tackling the international context in which the reform has been conceived and developed. It then presents and comments the process of change started after the approval of the 2006 Education Act and the main characteristics of the new model adopted. Finally, arguments in favour of considering this reform as unfinished are presented including an analysis of its main limitations and possible ways ahead.

KEY WORDS: educational reform, initial teacher training, teaching profession, European space for higher education.

\section{$* * * * *$}

\section{INTRODUCCIÓN}

El sistema educativo español viene asistiendo durante la última década a un proceso sostenido de cambios, que no parece haber llegado todavía a su punto final. Tras haberse completado en el año 2000 las transferencias educativas a las comunidades autónomas comenzó un proceso de transformaciones internas, tanto a escala autonómica como central, que ha afectado a muy diversos ámbitos del sistema educativo. Con mayor o menor amplitud e intensidad, se reorganizó la red de centros docentes, se modificó el modelo de dirección y gestión, se retocó la estructura del sistema y su ordenación, se reformuló el currículo de las diversas etapas, se revisó el modelo de orientación y apoyo al alumnado, se ofrecieron nuevas vías y oportunidades formativas a los estudiantes y se pusieron en marcha programas de cooperación territorial, por no citar sino algunos aspectos relevantes. Todo ello confluyó en una nueva regulación legal, compuesta fundamentalmente por la Ley Orgánica de Educación (LOE), promulgada en 2006, y varias leyes autonómicas, entre las que destacan la Ley de Educación de Andalucía (2007) y la de Cataluña (2009), que se encuentran actualmente en pleno desarrollo, así como las de Cantabria (2008) y Extremadura (2009). No obstante, la presentación de una nueva propuesta de reforma educativa por parte del gobierno hace pensar que seguimos en etapa transitoria, sin llegar todavía a alcanzar un punto de equilibrio. 
Como resulta fácil de entender, la formación del profesorado, tanto la inicial como la permanente, también se han visto afectadas por este conjunto de cambios. En líneas generales, puede decirse que la transformación principal consistió en la sustitución del modelo establecido por la Ley General de Educación de 1970, cuya manifestación más emblemática fue el Curso de Aptitud Pedagógica (CAP), por el actual modelo basado en la adecuación al sistema de grados y postgrados del Espacio Europeo de Educación Superior (EEES). Cabe recordar que, en la transición entre ambos, la Ley Orgánica de Ordenación General del Sistema Educativo (LOGSE) de 1990 y la Ley Orgánica de Calidad de la Educación (LOCE) de 2002 realizaron sendas propuestas intermedias, que no llegaron sin embargo a su plena aplicación. En realidad, se puede afirmar que el cambio más decisivo llegó con la reforma emprendida en 2007, como consecuencia del desarrollo de la LOE y su adaptación a las previsiones de la entonces recién reformada Ley Orgánica de Universidades (LOU).

Es precisamente de este último modelo del que tratan estas páginas. Dado que en este mismo monográfico Antonio Viñao aborda los principales modelos históricos de formación inicial del profesorado de educación secundaria en España, no tendré necesidad de echar la vista atrás. No prescindiré, en cambio, de adoptar una perspectiva comparativa, por cuanto considero que no se puede comprender cabalmente el sentido de los cambios en España al margen de lo que sucede en nuestro entorno más cercano.

Quiero dejar sentada también desde el comienzo la posible ambivalencia en que puedo incurrir al realizar este análisis y que estimo necesario que el lector conozca. Tengo la intención de efectuar un análisis crítico del modelo actual, pues comparto la opinión que lo considera incompleto y con serias limitaciones. Pero no se puede ignorar que estuve personalmente implicado en la toma de decisiones que llevó a su diseño y posterior puesta en marcha (TIANA, 2009). Como quiera que no pueda evitar esa duplicidad, creo que es necesario que el lector la conozca, como exigen las reglas del trabajo científico. No aspiro a lograr la objetividad absoluta, si es que tal cosa resultase posible, pero sí querría moverme en el terreno debidamente acotado de la intersubjetividad con el lector. Espero lograr al menos tal objetivo.

\section{EL CONTEXTO INTERNACIONAL: UN INTERÉS RENOVADO POR EL PROFESORADO Y SU FORMACIÓN}

Al igual que sucede en España, los sistemas educativos de la mayoría de los países occidentales llevan tiempo en proceso de transformación. Lo realmente nuevo en las últimas décadas es la proliferación de proyectos de reforma, de distinto alcance y propósito, promovidos con la intención de dirigir los procesos de cambio en los sistemas educativos (PEDRÓ y PUIG, 1998; HARGREAVES y SHIRLEY, 2009).

El foco de dichos procesos de transformación ha sido cambiante. Durante la década de los noventa se puso el énfasis sobre todo la transformación del currículo y la descentralización de la gestión educativa. A finales de la década se insistía más bien en elementos como el establecimiento de estándares y la puesta en marcha de sistemas de evaluación del rendimiento. En tiempos más recientes se ha insistido en aspectos tales como los nuevos modos de aprendizaje y los entornos que 
lo favorecen, el desarrollo de competencias básicas por parte de los estudiantes o la aplicación sistemática de las TIC en el espacio escolar. Y todo ello en un contexto de preocupación generalizada por la mejora de la calidad de la educación, convertida ahora en la principal fuente de justificación de las nuevas reformas (MARCHESI, TEDESCO y COLL, 2009).

Sin dejar de lado las orientaciones mencionadas, la primera década del siglo XXI asiste a un interés renovado por el papel que desempeña el profesorado en los procesos de cambio. A ello ha contribuido la nueva mirada que los estudios internacionales de evaluación a gran escala han arrojado sobre los sistemas educativos actuales. La expansión de programas como PISA, con voluntad expresa de incidir sobre las políticas educativas nacionales, ha planteado la necesidad de buscar explicaciones más refinadas del rendimiento de los estudiantes, sin limitarse a trazar el mapa de los resultados obtenidos (LUZÓN y TORRES, 2009; FERRER, 2012). Pero hoy sabemos que la explicación del rendimiento, delimitando los factores que en él inciden y su peso relativo, es una tarea ciertamente compleja, que está lejos de haber arrojado los resultados prometidos (TIANA, 2010). Ello ha llevado a desarrollar estudios con la intención de explorar en detalle algunos campos relacionados con el rendimiento de los sistemas educativos. Y entre todos ellos destaca con especial relevancia el que se refiere a la actuación del profesorado. Puede afirmarse que el profesorado está siendo objeto de un renovado estudio y análisis acerca de su preparación, su desempeño, las condiciones en que ejerce su profesión y su papel e inserción en el conjunto del sistema educativo.

Una buena demostración del interés suscitado en la última década por el estudio de la situación y las condiciones del profesorado se encuentra en la realización de sendos estudios a cargo de la OCDE y la red Eurydice de la Unión Europea, que han sido objeto de análisis interesantes (VV.AA., 2006).

El primero de dichos informes (OECD, 2005) fue el resultado de un proyecto desarrollado entre 2002 y 2004 en el que participaron 25 países. El estudio tenía como objetivo analizar varios aspectos: las principales tendencias y avances en la profesión docente; la evidencia disponible acerca de los factores considerados clave para atraer, desarrollar y retener a buenos docentes; las mejores políticas y prácticas aplicadas en este campo; las opciones políticas que cabría considerar; y las prioridades que deberían establecerse para el futuro. Entre las preocupaciones manifestadas por los países participantes se mencionaban las relativas al atractivo de la profesión docente, el desarrollo de los conocimientos y las habilidades del profesorado, los modelos de reclutamiento y selección de los docentes, y la capacidad de retener a los mejores docentes en el sistema educativo. Entre las orientaciones políticas más comunes, el estudio destacaba el énfasis en la calidad del profesorado sobre su cantidad, el desarrollo de perfiles docentes adecuados a las necesidades de las escuelas, la concepción de la carrera profesional como un continuo, la flexibilización de la formación y el acceso a la función docente, la transformación de la docencia en una profesión rica en conocimiento, y la concesión a los centros educativos de mayores responsabilidades para la gestión de su personal. El informe puso de relieve la necesidad de desarrollar políticas integradas de profesorado, que sobrepasarían los estrechos límites de la formación docente, por importante que esta sea. 
El segundo estudio fue en realidad un conjunto de informes publicados asimismo entre 2002 y 2005 por la red Eurydice de la Unión Europea, con el título genérico La profesión docente en Europa: Perfil, tendencias y problemática (EURYDICE, 2002-2005).A través de cinco volúmenes se pasaba revista a varios aspectos relativos a la profesión docente (I: Formación inicial y transición a la vida laboral; II: Oferta y demanda; III: Condiciones laborales y salarios; IV: El atractivo de la profesión en el siglo XXI; V: Reformas de la profesión docente: análisis histórico, 1975-2002). El conjunto del estudio estaba centrado en la educación secundaria inferior, por tratarse de la etapa educativa en que existe una mayor diversidad de situaciones de unos países a otros. Se trataba de un estudio comparativo sistemático y riguroso, que ofrecía una información de primera mano para entender los principales desafíos existentes en este ámbito (ESTEVE, 2006).

La publicación de ambos informes, el primero más centrado en el análisis de las políticas educativas y el segundo en la recopilación sistemática de la información disponible, ponía de manifiesto el renovado interés que se planteaba en torno a la profesión docente en los primeros años del siglo. Y esa tónica se mantendría en años posteriores, como veremos a continuación.

En el año 2007 se publicaba un estudio realizado por la consultora McKinsey \& Company que alcanzó una gran difusión y ha sido objeto de abundantes referencias. Con el título How the world's best-performing school systems come out on top se propuso analizar cómo los países que logran un mejor rendimiento educativo han llegado a conseguirlo (BARBER y MOURSHED, 2007). A partir de un estudio de la literatura existente, la realización de un conjunto de entrevistas con informadores relevantes en los países identificados y del análisis de algunos casos nacionales destacados, los autores buscaron la respuesta más convincente al interrogante planteado. Para ello se centraron en los factores de carácter sistémico, dejando al margen otros de naturaleza pedagógica o curricular, aun reconociendo su importancia.

El informe identifica la calidad del profesorado como el factor que tiene una mayor incidencia en los resultados obtenidos por los estudiantes, lo que se aprecia sobre todo en las primeras etapas escolares. La formulación de esa conclusión en forma de mensaje fácilmente comprensible ha alcanzado una gran difusión: "la calidad de un sistema educativo no puede ser superior a la calidad de su profesorado". Según el estudio, las prácticas en que coinciden los países que logran un mejor rendimiento son las siguientes: a) consiguen atraer a la docencia a los mejores estudiantes; b) los convierten después en unos buenos docentes; y c) aseguran que todos los alumnos reciban una enseñanza de gran calidad. Esa última práctica sirve de base para formular los otros dos mensajes centrales del estudio: "el único modo de mejorar el rendimiento consiste en mejorar la instrucción" y "lograr un alto rendimiento exige que todos los estudiantes alcancen el éxito escolar".

De acuerdo con el informe, disponer de los mejores docentes sería el resultado de dos estrategias complementarias: por un lado, seleccionar a los mejores estudiantes para que sean profesores; y por otra, pagarles un buen salario inicial como docentes. Para lograr el primer objetivo, el informe considera decisivo que la selección se realice con carácter previo a la formación inicial, lo que ofrece dos ventajas importantes: en primer lugar, aumenta el estatus de los estudios de profesorado, al hacer más exigente el acceso; en segundo lugar, permite utilizar mejor los 
recursos disponibles, al disminuir el número de estudiantes. Esa decisión debería además acompañarse de la exclusión de aquellos estudiantes que, una vez comenzados sus estudios, no demuestren las habilidades necesarias para ejercer la docencia. Como se dice provocativamente en el informe, "una mala decisión de selección puede resultar hasta en 40 años de mala enseñanza".

Esta primera conclusión plantea varios desafíos para las políticas de formación inicial del profesorado. Son muchos los sistemas educativos que, al igual que el español, no seleccionan a los estudiantes que quieren acceder a carreras orientadas a la docencia. Se acepta a un número mucho mayor de estudiantes que los puestos de profesores realmente disponibles y posteriormente se aplican sistemas rigurosos de selección para acceder a un puesto de trabajo. En opinión de los autores del informe, ese mecanismo conduce a la frustración de muchos docentes potenciales, a la falta de atractivo para los mejores estudiantes y a un gasto indiscriminado de recursos, al tener que formar a personas que luego no podrán ejercer la profesión.

Tras acceder a la docencia, el desafío consiste en mejorar de manera continua la enseñanza impartida por los nuevos profesores. En el informe se subraya la necesidad de que los docentes lleguen a ser conscientes de sus debilidades, conozcan las mejores prácticas y su sentido y estén motivados para introducir las mejoras necesarias. Esos objetivos se pueden alcanzar a través de medios como el desarrollo de habilidades prácticas durante la etapa de formación inicial, el establecimiento de tutorías para apoyar al profesorado en su trabajo docente, la formación de líderes instructivos eficaces y la oferta de posibilidades para que los profesores puedan aprender de otros docentes.

Esta segunda conclusión plantea asimismo varios desafíos para la formación inicial del profesorado, pero sobre todo para la formación permanente. Como puede apreciarse, plantea la necesidad de desarrollar actuaciones que superen la simple realización de cursos de formación, apostando más bien por reforzar el aprendizaje en el ejercicio profesional y propiciar la participación en centros educativos convertidos en organizaciones capaces de aprender. Y todo ello sin olvidar que la calidad del profesorado no puede interpretarse solamente en clave de su formación, sino también de las oportunidades existentes para lograr un verdadero desarrollo profesional.

En el año 2010 se publicaba un nuevo informe a cargo de McKinsey \& Company, con el título How the world's most improved school systems keep getting better (MOURSHED, CHIJIOKE y BARBER, 2010).En este caso se intentaba analizar la mejora lograda por veinte sistemas educativos seleccionados, descendiendo al estudio de los factores generales y específicos que explican sus avances. Se trata de un estudio con una metodología más sofisticada que el anterior, cuyo propósito es "ver el bosque sin perderse en los árboles", según la expresión utilizada por los propios autores.

El estudio agrupa a los países en cinco categorías, de acuerdo con su rendimiento en PISA: pobre, suficiente, bueno, muy bueno y excelente. Se trata de sistemas educativos con diferentes niveles de rendimiento, pero que en todos los casos han avanzado al menos de un escalón al siguiente, demostrando así que cualquier sistema es susceptible de mejora independientemente de 
cuál sea su nivel de partida. El estudio se propone analizar los principales factores que explican ese avance, identificando las intervenciones y las condiciones necesarias para iniciar el proceso de mejora, así como los mecanismos que han permitido su sostenibilidad a lo largo del tiempo.

Según dichos análisis, el factor clave para lograr el avance de los niveles medianos a los superiores (si bien no es exactamente así en el caso de los inferiores) consistiría en lo que denomina "dar forma a la profesión docente". Esta formulación tiene varias implicaciones. En primer lugar, se trataría de aumentar la valía de los nuevos docentes, mediante la elevación del nivel de entrada de los candidatos y la mejora de la calidad de su formación. En segundo lugar, se debería mejorar el desarrollo profesional del profesorado, aumentar sus oportunidades de aprendizaje de sí mismo y de los otros, establecer tutorías o sistemas de asesoramiento o mentoría (coaching) que permitan a los profesores mejorar sus habilidades de manera continua y ofrecer una carrera profesional con los incentivos adecuados. En tercer lugar, habría que fomentar la autoevaluación de los docentes y de los centros educativos y dar flexibilidad a estos últimos para organizarse de acuerdo con sus necesidades.

En estos últimos años, la OCDE ha realizado otros dos estudios adicionales acerca del profesorado, que también merece la pena destacar. En 2008 se llevó a cabo el estudio Teaching and Learning International Survey (TALIS), encaminado a conocer las prácticas de la enseñanza y el aprendizaje desde la perspectiva del profesorado y de la dirección escolar (OECD, 2009; Instituto de Evaluación, 2009). El estudio se basó en la aplicación de cuestionarios a una muestra de docentes y directores de centros de educación secundaria inferior en un total de 24 países, aunque finalmente se desecharon los de uno de ellos por su insuficiente tasa de respuesta. Se trata, por lo tanto, de un estudio empírico, a diferencia de los anteriormente mencionados. A partir de esos datos, la Comisión Europea encargó un estudio acerca de la situación de los países europeos, que complementa la información procedente de la OCDE con otras fuentes (EUROPEAN COMMISSION, 2010).

El informe resultante está centrado en varios aspectos relativos a la profesión docente, tal como es percibida por los encuestados. Entre ellos sobresalen los siguientes: la atención a las necesidades de desarrollo profesional manifestadas por los docentes, la participación del profesorado en la oferta existente y los medios más efectivos; las prácticas docentes, las actividades profesionales del profesorado, sus ideas acerca de la enseñanza y el aprendizaje, y la autopercepción de su eficacia y su satisfacción en el trabajo; la evaluación del profesorado y el retorno de la información generada por ese medio; los estilos de dirección escolar y su relación con el desarrollo profesional de los docentes; y la situación de dos características fundamentales de un entorno escolar positivo, como son el clima escolar y la autoeficacia docente. La publicación resulta de gran interés para conocer la opinión del profesorado acerca de diversos aspectos relativos a la profesión docente, incluida su formación permanente.

Más recientemente, en marzo de 2011, la OCDE convocó la primera Cumbre Internacional sobre la Profesión Docente, que dio lugar a una interesante publicación (OECD, 2011). El informe, que recoge la evidencia existente acerca de los temas que se iban a tratar en la reunión junto a sus principales conclusiones, se centra en cuatro grandes temas: el reclutamiento y la formación inicial 
del profesorado; el desarrollo profesional, el apoyo, las condiciones de empleo y la carrera de los docentes; la evaluación docente y los incentivos al profesorado; y la implicación del profesorado en la reforma educativa. Entre sus conclusiones principales cabe subrayar las siguientes: a) disponer de unos docentes de alta calidad es el resultado de decisiones políticas deliberadas y cuidadosamente desarrolladas a lo largo del tiempo; b) hacer de la enseñanza una profesión atractiva y eficaz requiere apoyar el aprendizaje permanente del profesorado, desarrollar estructuras de carrera que otorguen nuevos papeles a los docentes, e implicarles como agentes de la reforma escolar y no solo aplicadores de planes diseñados por otros; c) las políticas de profesorado no deben ser parciales, sino que han de adoptar un enfoque global; d) el desarrollo profesional ha de integrarse no solo en la carrera individual del docente, sino en el proceso de los cambios escolares y del sistema educativo, pues está demostrado que los modelos colaborativos de reforma educativa pueden ser muy eficaces.

En conjunto, todos estos informes plantean diversos elementos de un programa comprensivo de actuación para la mejora escolar, que tiene implicaciones importantes para la formación inicial y continua del profesorado. Se puede estar de acuerdo o en desacuerdo total o parcial con sus conclusiones más destacadas, pero no cabe duda de que vienen a subrayar varios elementos que resultan pertinentes para analizar las políticas actuales de formación del profesorado.

\section{LA REFORMA RECIENTE DE LA FORMACIÓN INICIAL DEL PROFESORADO EN} ESPAÑA

Si los estudios citados han puesto de manifiesto que en los últimos años se ha ido extendiendo un amplio acuerdo acerca de la necesidad de revisar la configuración general de la profesión docente, la mayor parte de ellos no pudieron ser tomados como fuente directa para la reciente reforma española. En efecto, dado que el proceso de transformación se puso en marcha en 2005 y se plasmó finalmente en la regulación normativa de 2007, esos informes no llegaron a tiempo de servir de fuente de inspiración, como hubiese sido deseable. Otra cosa puede decirse sin embargo de los trabajos desarrollados en el seno de la Unión Europea, que influyeron más directamente en las decisiones adoptadas.

\subsection{El marco europeo: políticas en materia de formación del profesorado}

Aunque tras la adopción del Acta única de 1986 las instituciones europeas comenzaron a interesarse por la educación en general y por la formación del profesorado en particular, fue a partir de la aprobación de la denominada Estrategia de Lisboa en el año 2000 cuando las primeras declaraciones y acciones pioneras dieron paso a una verdadera política de educación y formación en el seno de la Unión Europea. Ese ambicioso plan de actuación para la primera década del nuevo siglo, aun respetando el principio de subsidiariedad, implicó la adopción de políticas coordinadas en materia de educación.

Concretamente, fue en los Objetivos precisos de los sistemas de educación y formación donde se contempló abiertamente la necesidad de reforzar la formación docente. En desarrollo de la Estrategia de Lisboa, el Consejo Europeo de Estocolmo aprobó en 2001 tres objetivos concretos 
para los sistemas de educación y formación, cada uno de los cuales incluía varios objetivos precisos. El primero de los objetivos concretos aprobados en dicha reunión planteó la necesidad de "Mejorar la calidad y la eficacia de los sistemas de educación y formación en la Unión Europea". Y el primero de los objetivos precisos incluidos en esa categoría consistió precisamente en "Mejorar la educación y la formación de profesores y formadores". En el texto que explicaba más extensamente dicho objetivo se planteaba la necesidad de que los conocimientos y las capacidades de los docentes respondiesen a los cambios y las expectativas de la sociedad en que se insertan y se adaptasen a la variedad de los grupos con los que trabajan. Al año siguiente, en el Consejo de Barcelona de 2002, se planteó la necesidad de que el profesorado fuese capaz de responder a las exigencias de la sociedad del conocimiento. Con ese propósito, se constituyó un grupo específico de trabajo dedicado a la mejora de la formación de los profesores y formadores, que contó con la participación de representantes de todos los países participantes en el Programa Educación y Formación 2010. Y en esas mismas fechas se puso en marcha la producción del amplio informe de Eurydice mencionado en el apartado anterior, que sirvió de marco para la reflexión y el debate que se estaba desarrollando.

El principal resultado de dicho grupo de trabajo fue la Comunicación de la Comisión al Consejo y al Parlamento Europeo de 3 de agosto de 2007, con el título Mejorar la calidad de la formación del profesorado. La Comunicación tenía por objetivo "evaluar la actual situación en la Unión Europea por lo que se refiere a la formación del profesorado y proponer una reflexión compartida de las medidas que pueden adoptarse en los Estados miembros y cómo podría apoyarlas la Unión Europea" (COMISIÓN DE LAS COMUNIDADES EUROPEAS, 2007: 2).

El documento responde a una doble convicción: por una parte, la profesión docente es cada vez más compleja y las exigencias al profesorado van en aumento; por otra, mejorar la calidad de la formación de los docentes debe considerarse un objetivo fundamental por parte de los sistemas educativos europeos. Para responder a los retos planteados, se proponen unos principios comunes para mejorar las competencias y la cualificación del profesorado. Dicha propuesta responde a una visión de la docencia basada en los siguientes principios:

- Alta cualificación: la docencia debe ser una profesión con un nivel de formación inicial al menos de Grado, cursada en instituciones de educación superior, en la que se desarrollen competencias que sirvan para adquirir conocimientos de las materias a impartir, formación pedagógica y didáctica, práctica en la investigación educativa como medio para la mejora de la actividad docente, capacitación en el uso de instrumentos y recursos para guiar y apoyar a los estudiantes, y conocimiento de la dimensión social y cultural de la educación.

- Aprendizaje permanente: debe estar enmarcada en el contexto del aprendizaje a lo largo de la vida, lo que implica prestar una atención especial al desarrollo profesional continuo, dotando al profesorado de nuevos conocimientos, destrezas y recursos que le permitan innovar en su trabajo.

- Movilidad: debe facilitarse la movilidad docente entre distintos países europeos, diferentes niveles educativos y distintas profesiones dentro del sector de la educación. 
- Colaboración: debe estar basada en la colaboración entre el profesorado, los centros docentes y los organismos sociales locales, regionales, estatales y comunitarios con competencias educativas y culturales.

Con esa perspectiva, la Comisión propuso la adopción de una serie de medidas políticas en este ámbito, entre las que cabe destacar las siguientes: a) considerar la formación y el desarrollo profesional de cada docente como una tarea permanente, estructurada y adecuadamente financiada; b) asegurar una continuidad ininterrumpida de formación que abarque la etapa inicial, la inducción a la docencia y el desarrollo profesional continuo; c) ofrecer la posibilidad a los docentes de adquirir en cualquier momento de su carrera toda la gama de conocimientos de las materias que imparten, así como las actitudes y capacidades pedagógicas necesarias; d) permitir a los docentes hacerse cargo de sus propios itinerarios de aprendizaje.

Este importante documento fue objeto de preparación y discusión al tiempo que se preparaba y lanzaba la reforma española, con lo que resultó posible tenerlo en cuenta en su diseño, especialmente en lo que se refiere a la formación inicial del profesorado. De hecho, además de la participación de representantes españoles en el grupo de trabajo, el documento resultante sirvió de base para negociaciones previas a la adopción de la nueva normativa.

\section{$\underline{\text { 2.2. El sentido y el proceso de la reforma }}$}

La aprobación de la LOE en el año 2006 supuso el comienzo formal del proceso de cambio de la formación inicial del profesorado. En sus artículos 92 y siguientes se sentaron las bases de la nueva regulación, al equiparar los títulos de Maestro de educación infantil y de educación primaria con los nuevos títulos universitarios de Grado y prever una formación pedagógica y didáctica de nivel de posgrado para el nuevo profesorado de las distintas ramas de la educación secundaria. Y en el artículo 100 se estableció la exigencia de poseer dichas titulaciones para poder ejercer la docencia. Hay que tener en cuenta que en ese momento aún no estaba concluida en España la reforma de los títulos universitarios adaptados al EEES, motivo por el cual resultó imposible avanzar más allá. Pero a pesar de dicho condicionante, esa formulación tuvo dos efectos decisivos: por una parte, estableció con claridad el nivel formativo exigido a los docentes de los diferentes niveles educativos, adelantándose incluso a la nueva regulación de los estudios universitarios y manteniendo la necesaria coherencia con los planteamientos europeos; por otra, permitió considerar la docencia como una profesión regulada, lo que autorizaba al Gobierno a establecer algunas condiciones para la aprobación de los títulos orientados a la formación de dichos profesionales. Ambas condiciones resultaron clave para entender el sentido de las decisiones que posteriormente se adoptaron.

No obstante, aun siendo cierto que la LOE representó el arranque de la nueva regulación normativa, no se puede olvidar que los primeros pasos se habían dado con anterioridad. Concretamente, hay que recordar que el Ministerio de Educación y Ciencia había lanzado en septiembre de 2004 el documento titulado Una educación de calidad para todos y entre todos, con objeto de promover un debate público acerca de la situación del sistema educativo español y los posibles cambios que podrían introducirse (MEC, 2004). Su capítulo 11, incluido bajo el epígrafe 
más amplio "El imprescindible protagonismo del profesorado", estaba específicamente dedicado a la "Calidad y valoración del profesorado". Allí ya se avanzaba la propuesta de que "la formación inicial del profesorado se adaptará al nuevo modelo de grados y postgrados del espacio europeo de educación superior" (MEC, 2004: 113). Como nos recuerda Antonio Moreno, director en esa época del Instituto Superior de Formación del Profesorado (ISFP), la propuesta "recibió una magnífica acogida por quienes a través del foro virtual participaron en la consulta, además de otras aportaciones llegadas al MEC por diversos conductos" (MORENO, 2009: 43-44).

La propuesta concordaba con otras que se habían realizado antes de aquella fecha. En concreto, conviene mencionar que en el año 2004 había visto la luz el denominado Libro Blanco sobre el Título de Grado en Magisterio, desarrollado por una amplia red de universidades, bajo la coordinación de la Universidad Autónoma de Madrid, y auspiciado por la Agencia Nacional de Evaluación de la Calidad y Acreditación (ANECA, 2004). En ese relevante documento se realizaba una propuesta similar a la finalmente incluida en la LOE para la formación inicial del magisterio.

A partir de la propuesta formulada en el documento ministerial de 2004, y todavía durante el proceso de elaboración y discusión de la nueva ley, el ISFP organizó varios seminarios acerca de la formación inicial del profesorado, con objeto de analizar los planes de estudio vigentes y proponer orientaciones para elaborar los nuevos grados y posgrados. En esas reuniones, llevadas a cabo durante el curso 2004-2005, participaron docentes de las diversas áreas de conocimiento implicadas en la formación docente. El proceso resultó muy provechoso, pues permitió conocer en detalle la situación existente, las expectativas manifestadas y las posibles vías de respuesta a las necesidades detectadas. La regulación posterior se basó en gran medida en el trabajo allí realizado.

El paso más importante que se dio a continuación consistió en la aprobación de las directrices para los nuevos títulos universitarios orientados a la formación del profesorado. En un primer momento se elaboraron las fichas técnicas de propuesta de dichos títulos, de acuerdo con lo establecido en un primer Real Decreto de 2005 que regulaba la nueva estructura de los estudios universitarios. Esas fichas fueron discutidas en el Consejo de Coordinación Universitaria y la Comisión General de Educación a mediados de 2006, recibiendo diversas observaciones y sugerencias. No obstante, la derogación de aquella norma y su sustitución por una nueva obligó a modificar las previsiones iniciales.

El fundamento legal de la nueva regulación, además de lo establecido en la LOE, se encuentra en el Real Decreto 1393/2007, que establece la ordenación de las enseñanzas universitarias oficiales ${ }^{1}$. En su artículo 12.9 establece que "Cuando se trate de títulos que habiliten para el ejercicio de actividades profesionales reguladas en España, el Gobierno establecerá las condiciones a las que deberán adecuarse los correspondientes planes de estudios, que además deberán ajustarse, en su caso, a la normativa europea aplicable". En desarrollo de esa disposición se publicaron tres resoluciones de la Secretaría de Estado de Universidades e Investigación, recogiendo otros tantos acuerdos de Consejo de Ministros de 14 de diciembre de 2007, por las que

\footnotetext{
${ }^{1}$ Se trata del Real Decreto 1393/2007, de 29 de octubre, por el que se establece la ordenación de las enseñanzas universitarias oficiales, publicado en el Boletín Oficial del Estado de 30 de octubre, que sustituyó al de 2005 y que se puede consultar en http://www.boe.es/boe/dias/2007/10/30/pdfs/A44037-44048.pdf
} 
se establecían las condiciones a las que deberían adecuarse los títulos que habilitasen para las profesiones de Maestro de Educación Infantil, Maestro de Educación Primaria y Profesor de Educación Secundaria(incluyendo la etapa obligatoria, el bachillerato y la formación profesional de

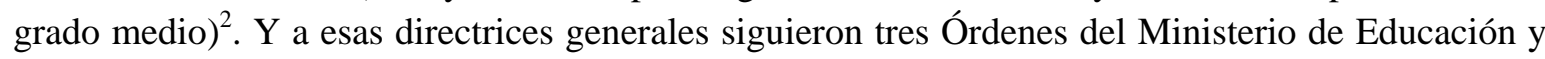
Ciencia en las que se establecían los requisitos para la verificación de los respectivos títulos universitarios que pudiesen elaborar las universidades ${ }^{3}$. En estas últimas se establecían las condiciones que deberían cumplir los títulos sometidos posteriormente a verificación por las agencias de acreditación ${ }^{4}$. Dichas condiciones incluían una relación de las competencias generales que los estudiantes deberían adquirir, algunos aspectos relativos a la planificación de las enseñanzas (como el número total de créditos, el nivel lingüístico que los estudiantes deberían demostrar, las características generales del Practicum o la posibilidad de menciones cualificadoras) y un anexo en el que figuran los bloques de contenidos formativos, con su número mínimo de créditos y los módulos que deben incluir, así como las competencias específicas correspondientes a cada uno de ellos.

Estos documentos constituyen la base en que se deben apoyar las universidades para elaborar sus propuestas de títulos orientados a la formación inicial del profesorado. En el año 2008 comenzaron a elaborarse las nuevas propuestas de títulos de grado de magisterio y de máster de profesorado de educación secundaria, que se sometieron al obligado proceso de verificación. Tras su aprobación por ANECA, comenzó un proceso paulatino de implantación de los nuevos títulos, que aún no ha finalizado completamente aunque está muy avanzado. A partir de este momento, el proceso de desarrollo de las titulaciones y su implantación, así como su seguimiento, corresponde a los centros universitarios respectivos, de acuerdo con las normas que regulan esos aspectos.

\subsection{Principales características del modelo adoptado}

El modelo así construido es el que está vigente en la actualidad. Vale la pena destacar sus principales rasgos distintivos, situándolos además en un contexto comparativo que permita entender sus semejanzas con otros modelos y sus peculiaridades. Entre sus características más sobresalientes destacan las siguientes.

\subsubsection{Adecuación al espacio europeo de educación superior (EEES.}

Como se ha señalado, los nuevos títulos de magisterio han pasado a ser títulos de Grado, cuya duración se ha establecido en 240 créditos. Además de adecuarse así a la nueva estructura de los títulos universitarios, también su duración total se equipara a la que Francesc Pedró señalaba como la más habitual entre los países miembros de la OCDE (PEDRÓ, 2006). Hay que recordar que a los maestros españoles se les exigía hasta entonces un título de Diplomado, de tres años de

2 Los textos pueden consultarse en http://www.boe.es/boe/dias/2007/12/21/pdfs/A52846-52847.pdf y http://www.boe.es/boe/dias/2007/12/21/pdfs/A52851-52852.pdf

${ }^{3}$ Pueden consultarse en http://www.boe.es/boe/dias/2007/12/29/pdfs/A53735-53738.pdf (Maestro educación infantil), http://www.boe.es/boe/dias/2007/12/29/pdfs/A53747-53750.pdf (Maestro educación primaria) y http://www.boe.es/boe/dias/2007/12/29/pdfs/A53751-53753.pdf (Profesor de educación secundaria).

${ }^{4}$ Una descripción detallada del proceso de delimitación de los requisitos exigibles para los nuevos títulos se encuentra en Moreno (2009). 
duración. Por lo tanto, la transformación de Magisterio en un título de Grado, de cuatro años, no hizo sino situar a España en condiciones similares a la mayoría de los países desarrollados. Además, esta decisión tiene una implicación importante, ya que los nuevos maestros (graduados) que hayan realizado un máster (postgrado) tienen la puerta abierta para acceder al doctorado, lo que no era anteriormente el caso. Las posibilidades que ofrece esta nueva configuración han sido bien valoradas.

También la formación del profesorado de educación secundaria se ha adecuado al EEES, al exigir un título de Máster, con una duración de 60 créditos. En este caso, el modelo español no concuerda exactamente con todos los cercanos, puesto que hay países que diferencian la formación del profesorado de educación secundaria inferior (la ESO en nuestro caso) y la del ciclo superior (nuestro bachillerato).En el caso español no existe esa distinción, si bien fue uno de los asuntos que generaron cierto debate en el proceso de elaboración de los nuevos títulos. Los docentes con esa formación pueden ejercer en diversas modalidades, cuyas enseñanzas se ubican además en los mismos centros, lo que no sucede en todos los países europeos. Por otra parte, la decisión de exigir un título específico de máster para acceder a la docencia en educación secundaria implica exigir al profesorado una formación inicial de cinco años, lo que sitúa a los docentes españoles de ESO entre los que tienen una formación más prolongada en el marco de la OCDE, aunque no sea el caso del profesorado de bachillerato (PEDRÓ, 2006).

2.3.2. Combinación de un modelo simultáneo para los maestros y otro consecutivo para los profesores de educación secundaria

La formación inicial de los nuevos maestros y profesores de educación secundaria sigue dos modelos diferentes en lo que respecta a la combinación de los estudios de diverso tipo (instrumental, disciplinar, pedagógico y didáctico) que deben realizar. Conviene recordar que existen a grandes rasgos dos modelos distintos de organización de dicha formación, el sucesivo y el simultáneo. En el primero, la formación propiamente orientada a la docencia viene precedida por una formación general en algún campo específico del conocimiento. En el segundo, ambos componentes se imbrican a lo largo del proceso formativo, con distintos grados de integración entre ambos.

En el caso de España, los modelos adoptados para formar maestros y profesores difieren entre sí. Los estudios de magisterio son de carácter simultáneo, mientras que la formación del profesorado de educación secundaria adopta el modelo sucesivo, completándose primero un grado en cualquier campo del conocimiento y realizando después un máster de formación pedagógica y didáctica. Se trata de una decisión que también ha suscitado algunas críticas, aunque es el modelo que cuenta con mayor tradición en España.

\subsubsection{Grado notable de apertura de la regulación general.}

Frente al modelo existente en España antes de la reforma de los estudios universitarios de 2007, que determinaba de manera muy precisa los planes de estudio para la formación de maestros o los contenidos del CAP, el nuevo modelo deja un amplio margen de libertad a las universidades 
para determinar sus propuestas de títulos y su posterior aplicación. Es cierto que el hecho de tratarse de profesiones reguladas ha permitido establecer al menos unos requisitos básicos para el proceso de verificación, cosa que existe en muy pocos campos profesionales. No obstante, las orientaciones establecidas en las órdenes ministeriales de 2007 son mucho menos amplias y precisas que las anteriormente existentes, e incluso que las propuestas en 2005, si bien no se trata de requisitos menos detallados que los establecidos para profesiones como la de arquitecto o ingeniero, existiendo una similitud grande en todos los casos. Por lo tanto, la situación permite un considerable grado de flexibilidad y de capacidad de actuación por parte de las universidades.

\subsubsection{Importancia concedida a la formación práctica}

El nuevo modelo concede una notable importancia a la formación práctica, considerándola un componente fundamental, entre otras razones, por estar claramente enraizada en la necesidad de construir una identidad profesional. En efecto, como señala Carlos Marcelo (2009a), el proceso de convertirse en docente supone adquirir, recuperar y gestionar conocimientos que tienen diferentes orígenes y formas de expresión, lo que implica tomar en consideración las distintas oportunidades de aprendizaje que se le ofrecen al futuro docente, no sólo las formales, sino también las informales. Para António Nóvoa (2009), la atención prioritaria a la práctica resulta capital para dar respuesta a la necesidad de formar buenos profesores. Su propuesta consiste en asegurar que la formación de profesores asume un fuerte componente práctico, centrado en el aprendizaje de los alumnos y en el estudio de casos concretos, teniendo siempre como referencia el trabajo escolar.

De acuerdo con estos planteamientos, la formación práctica adquiere una gran importancia en el nuevo modelo español de formación inicial docente. No se trata simplemente de una fase de aplicación de los conocimientos teóricos previamente adquiridos, sino de un verdadero proceso formativo, que requiere un espacio propio en el que se incorporen todos aquellos conocimientos y competencias que requieren una reflexión a partir de la experiencia práctica. Así se ha concebido el Practicum al que se refieren las órdenes ministeriales de 2007, que debe desarrollarse en centros educativos de las etapas correspondientes.

\subsubsection{Carácter generalista de los títulos}

Los títulos de magisterio poseen un carácter generalista y su número se ha reducido drásticamente en relación con las siete especialidades que existían desde 1991. Siguiendo la propuesta del Libro Blanco de 2004 antes mencionado, los nuevos títulos se han reducido a dos: maestro de educación infantil y maestro de educación primaria. Cada uno de ellos debe preparar a los docentes que trabajarán en dichas etapas, huyendo de la anterior especialización, que muchos consideraban excesiva. Ahora bien, teniendo en cuenta la necesidad de atender a algunas enseñanzas que requieren una formación especializada, la nueva regulación contempla la posibilidad de establecer menciones cualificadoras. El sentido de esa propuesta es el siguiente: dado que los títulos han aumentado su duración, debiera ser posible mantener un fuerte núcleo generalista pero dejando abierto un cierto espacio de especialización, que puede implicar entre 30 y 60 créditos. Las menciones no forman una relación cerrada, sino que cada universidad puede ofrecer aquellas que considere más convenientes y tengan mayor demanda. 
Por otra parte, los requisitos aprobados para los nuevos títulos de máster no incluyeron finalmente una relación de especialidades, aunque sí existan para los cuerpos docentes de la educación secundaria. En consecuencia, la situación es bastante heterogénea, pues las especialidades ofrecidas por las universidades en sus másteres correspondientes se corresponden a veces exactamente con algunas especialidades docentes, mientras que en otros casos agrupan varias en una sola. Esa diversidad ha generado alguna incertidumbre, pero no tiene efectos legales para el acceso a la docencia, pues lo que se exige es simplemente haber cursado dicho máster, sin especificar en qué especialidad. En última instancia, estamos ante una muestra más del amplio margen de maniobra de que disponen las universidades para configurar su oferta académica, incluida la eventual selección de sus estudiantes.

\section{VALORACIÓN GENERAL: UNA REFORMA INCOMPLETA}

Hasta aquí se ha presentado el modelo reformado de formación inicial del profesorado español, situándolo en su contexto nacional e internacional. Pero el artículo quedaría incompleto si no se descendiese a una valoración del mismo, que por otra parte se adelanta en el propio título. Se habla allí de una reforma incompleta y habrá que justificar debidamente esa apreciación.

Desde mi punto de vista, considero que se puede hablar de un proceso incompleto, y nótese bien que no digo frustrado, porque pienso que aún estamos a tiempo de reorientar algunos de sus puntos más problemáticos. En términos generales, comparto la apreciación globalmente positiva de Antonio Moreno, cuando afirma que la formación inicial del profesorado "sin duda, ha dado un paso histórico esencial para situar a maestros y profesores en la posición de dignidad académica y de prestigio profesional muchas veces deseada y reclamada pero, hasta ahora, nunca conseguida" (MORENO, 2009: 43). Pero ello no quita para que debamos reconocer que existen aspectos criticables 5 .

Los problemas que me parecen más importantes y sobre los que vale la pena reflexionar se agrupan en dos grandes categorías. Por un lado, hay algunas limitaciones derivadas del propio modelo de formación adoptado y del modo en que se ha desarrollado. Por otra parte, hay otras derivadas del hecho de haber reformado la formación inicial docente, sin haberlo hecho al mismo tiempo con otros componentes de la profesión docente. Veamos con mayor detenimiento ambos grupos de cuestiones.

Lo primero que cabe destacar es que el modelo adoptado, que deja gran capacidad de decisión a las universidades para la propuesta de títulos adaptados al EEES, tendría que equilibrarse con una mayor interacción con el conjunto del sistema educativo, que no se ha producido en medida suficiente. En efecto, cuando hablamos de formar docentes para la educación infantil, primaria y secundaria debemos tener en cuenta cuáles son las necesidades y las demandas propias de esos niveles educativos. La universidad tiene la misión de formar personas capaces de desarrollar una actividad profesional, para lo que requiere que adquieran unos instrumentos intelectuales y prácticos. Pero el desarrollo de las capacidades y competencias necesarias no puede producirse en el

\footnotetext{
${ }^{5}$ Uno de los pocos intentos que conozco de abordar una evaluación sistemática y rigurosa de la aplicación del nuevo modelo, para el caso del profesorado de educación secundaria, se encuentra en Manso (2012).
} 
vacío, al margen de cuál será su trabajo profesional. De ahí deriva precisamente la consideración de la docencia como profesión regulada, que es lo que ampara el establecimiento de algunos criterios que debe cumplir los planes de estudio universitarios.

Pues bien, hay que reconocer que en la mayor parte de los casos la planificación se ha realizado exclusivamente en el ámbito universitario, sin contar con los profesionales e instituciones destinatarias de los nuevos docentes. Han sido pocas las comunidades autónomas que han planificado, por ejemplo, la oferta de plazas para los nuevos títulos teniendo en cuenta la posibilidad de ofrecer una buena formación práctica, y menos aún las que han utilizado este mecanismo para incentivar la mejora de los centros receptores de los futuros docentes. Es necesario señalar que una buena concepción de la formación práctica puede producir un efecto beneficioso para reforzar la conexión entre las instituciones de formación del profesorado y los centros de educación infantil, primaria y secundaria, integrando a estos últimos en una perspectiva amplia y capaz de abrir espacios nuevos para el desarrollo profesional. La participación en tareas formativas puede servir de estímulo para reforzar las buenas prácticas docentes, de medio para reconocer la organización y el buen funcionamiento de los centros seleccionados como centros de prácticas y de elemento de vinculación de su profesorado con las instituciones universitarias.

Ese componente práctico permitiría además replantear la selección del alumnado candidato al profesorado, considerada un elemento central para el refuerzo de la profesión docente. En efecto, hay que reconocer que limitar las plazas de alumnado a las necesidades previstas de nuevos docentes no resulta sencillo en sistemas universitarios como el español, puesto que la exigencia social de ofrecer un número de plazas universitarias en proporción similar a la demanda y el beneficio económico que obtienen las universidades por contar con más alumnado juegan en contra de la contención de la oferta. Por ese motivo, la única posibilidad real de limitar de manera justa el número de estudiantes en estas titulaciones consistiría en ponerla en conexión con la previsión de nuevas plazas docentes y con la exigencia de proporcionar al nuevo profesorado una buena formación práctica. Profundizar en esta dirección quizás podría suponer un revulsivo importante para desarrollar un modelo de formación inicial del profesorado más riguroso, exigente y prestigioso.

A este respecto, cabe señalar que en los últimos tiempos se ha esbozado la idea de establecer un sistema de prácticas para el profesorado en formación similar al establecido para otras profesiones, como es el caso de las especialidades médicas. Se trataría de configurar el proceso de formación práctica como un continuo que comenzase con intervenciones sencillas y fuese procediendo a otras más complejas, hasta llegar a lograr plena autonomía profesional. Ese modelo implicaría determinar y programar de manera sistemática las áreas y el tipo de intervención que correspondería a cada escalón formativo. También exigiría diseñar un sistema de tutoría y mentoría (coaching), que incluso podría prolongarse más allá de la etapa propiamente formativa.

Este modelo podría ofrecer dos posibilidades interesantes. En primer lugar, permitiría suavizar la rigidez del actual modelo consecutivo de formación del profesorado de educación secundaria, tendiendo a integrar en mayor medida sus dos fases. Vale la pena subrayar a este respecto que el peso del modelo universitario tradicional en España ha influido notablemente en la 
imposibilidad de crear un título de Grado específicamente orientado a la docencia en la educación secundaria, como existe en otros países y pese al apoyo a esta idea por parte de especialistas reconocidos (ESTEVE, 2009). Y también conviene recordar el rechazo que ese planteamiento recibió en el proceso de reforma de los planes de estudio universitarios de los años noventa, así como la contestación que sufrió el nuevo máster de formación docente por parte de algunos sectores académicos que reprochaban que dicho complemento de formación fuese de carácter pedagógico y no exclusivamente disciplinar. En segundo lugar, este modelo permitiría replantear el proceso de inducción a la docencia, que tanta atención está recibiendo en la actualidad. El comienzo del ejercicio profesional es difícil, tanto por motivos puramente laborales, como de carácter emocional o derivados de la significación social de la enseñanza. Por ese motivo, es cada vez más frecuente oír a especialistas que propugnan la existencia de una etapa formalizada de inducción a la profesión docente (MARCELO, 2009b). Es cierto que la LOE previó el seguimiento de los profesores noveles durante su primer año de ejercicio profesional, asignándoles un tutor a esos efectos. Sin embargo, aunque la norma está correctamente concebida, hay que reconocer que se encuentra todavía lejos de haberse trasladado a la práctica. A pesar de la importancia concedida a la reforma de la formación inicial del profesorado, no se ha completado con un modelo eficaz de inducción a la docencia, que está aún por diseñar y desarrollar.

Pero quizás mayor importancia que los problemas mencionados hasta el momento tenga la ausencia de una mayor conexión entre la formación inicial del profesorado y otros elementos fundamentales de la profesión docente. Como se ha podido apreciar, la mejora de la calidad del trabajo docente constituye en la actualidad una preocupación de primer orden para todos los sistemas educativos. Los estudios mencionados más arriba subrayan la importancia que tiene el desarrollo de políticas articuladas de profesorado que permitan contar con docentes bien formados e incentivados, capaces de seguir aprendiendo a lo largo de su carrera, dispuestos a colaborar con sus compañeros y a trabajar en equipo y comprometidos con la mejora de la calidad de la enseñanza que imparten. La agenda política en este campo debe tener en cuenta todas esas dimensiones, lo que la hace ciertamente exigente.

Existe un acuerdo bastante generalizado acerca de la necesidad de desarrollar dos tipos de políticas confluyentes en este ámbito. Por una parte, es necesario adoptar políticas que se dirijan a la profesión docente en conjunto, con el objetivo de mejorar su estatus, su productividad, su desarrollo profesional y la calidad del entorno escolar. Por otra parte, hay que adoptar políticas orientadas a atraer y retener a profesores de determinadas áreas y materias, así como a los que trabajan en entornos especialmente difíciles. Ambas orientaciones políticas coinciden en la necesidad de contar con los mejores docentes, siendo la única diferencia entre ellas el hecho de que se orienten hacia el conjunto del sistema educativo o hacia algunos sectores específicos. Los objetivos generales podrían formularse en ambos casos de un modo similar: hacer de la enseñanza una opción profesional atractiva; desarrollar el conocimiento y la habilidad que poseen los docentes; reclutar, seleccionar y emplear a buenos profesores; retener a los docentes eficaces; y desarrollar unas buenas políticas de profesorado, que tengan bases sólidas e impliquen al profesorado en su diseño y aplicación. 
Como puede apreciarse, se trata de llevar a cabo políticas que aborden el conjunto de la profesión docente y no se limiten a la formación inicial del profesorado. Se puede achacar a la LOE que haya emprendido la reforma de la formación del profesorado en España sin haber abordado otros aspectos tales como el acceso a la carrera docente, los incentivos a lo largo de la misma y los medios para su mejora, lo que produce el efecto de dejar incompleto el proceso de reforma. Sin duda, se trata de una crítica justa, a la que sólo puede responderse planteando la relativa disociación de políticas y organismos responsables que existe en el ámbito del profesorado, sin que esa explicación la justifique. Como he puesto de relieve en otros lugares (TIANA, 2011), cualquier política que hoy se emprenda para mejorar la profesión docente no puede desligar la vertiente de la formación de otras vertientes relativas al acceso, la inducción a la docencia y el desarrollo profesional posterior. Se trata de dimensiones estrechamente entrelazadas, sobre todo si se contempla el efecto práctico que tienen las políticas adoptadas.

Por todos estos motivos, creo que se puede legítimamente hablar de una reforma incompleta, por importantes que hayan sido los pasos dados. No obstante, no creo que estemos en una situación sin salida, sino que más bien considero que se ven en el horizonte varios caminos de avance sobre los que valdría la pena reflexionar y debatir.

\section{REFERENCIAS BIBLIOGRÁFICAS}

ANECA (2004): Libro Blanco Título de Grado en Magisterio (Madrid, ANECA) 2 vols., (http://www.aneca.es/var/media/150404/libroblanco_jun05_magisterio1.pdf), consultado el 5 de noviembre de 2012.

BARBER, M. \& MOURSHED, M. (2007): How the world's best-performing school systems come out on top (McKinsey\&Company) [Consultado el 1 de diciembre de 2010], (http://www.mckinsey.com/App_Media/Reports/SSO/Worlds_School_Systems_Final.pdf)

COMISIÓN DE LAS COMUNIDADES EUROPEAS (2007): Mejorar la calidad de la formación del profesorado (Comunicación de la Comisión al Consejo y al Parlamento Europeo), Bruselas [Consultado el 1 de diciembre de 2010], (http://eurlex.europa.eu/LexUriServ/LexUriServ.do?uri=COM:2007:0392:FIN:ES:PDF)

ESTEVE, J. M. (2006): La profesión docente en Europa: perfil, tendencias y problemática. La formación inicial. Revista de Educación, 340, pp. 19-40.

ESTEVE, J.M. (2009): Políticas de formación inicial y continua (maestros y secundaria). Crítica y propuestas. En M. de PUELLES BENÍTEZ,coord., Profesión y vocación docente. Presente y futuro (Madrid, Biblioteca Nueva - Escuela Julián Besteiro) pp. 139-162.

EUROPEAN COMISSION (2010): Teachers' Professional Development - Europe in international comparison - An analysis of teachers' professional development based on the OECD's Teaching and Learning International Survey (TALIS)(Luxembourg, Office for Official Publications of the European Union).

EURYDICE (2002-2005): La profesión docente en Europa: Perfil, tendencias y problemática, 5 volúmenes (Madrid, Secretaría General Técnica - CIDE) 
FERRER, F., (Coord.) (2012): PISA: Aportaciones e incidencias sobre las políticas educativas nacionales (monografía). Revista Española de Educación Comparada, 19.

HARGREAVES, A. \& SHIRLEY, D. (2009). The Fourth Way. The inspiring Future for Educational Change (Thousand Oaks, CA, Corwin).

INSTITUTO DE EVALUACIÓN (2009). TALIS (OCDE). Estudio Internacional sobre la Enseñanza y el Aprendizaje. Informe español 2009 (Madrid, Ministerio de Educación Instituto de Evaluación).

LUZÓN, A. y TORRES, M. (2009): PISA en el contexto de las evaluaciones internacionales. En M.A. PEREYRA et al, eds., PISA under Examination: Changing Knowledge, Changing Tests and Changing Schools / PISA a Examen: cambiar el conocimiento, cambiar las pruebas y cambiar las escuelas (Granada, Comparative Education Society in Europe CESE) pp. 11-41.

MANSO, J. (2012): La formación inicial del profesorado de Educación Secundaria: Análisis y valoración del modelo de la LOE. Tesis doctoral inédita. Universidad Autónoma de Madrid.

MARCELO, C. (2009a): Formalidad e informalidad en el proceso de aprender a enseñar. Revista de Educación, 350, pp. 31-55.

MARCELO, C. (2009b). El profesorado principiante. Inserción a la docencia (Barcelona, Octaedro).

MARCHESI, A., TEDESCO, J.C. y COLL, C., (coords.) (2009): Calidad, equidad y reformas en la enseñanza (Madrid, Fundación Santillana-OEI).

MEC (2004): Una educación de calidad para todos y entre todos. Propuestas para el debate (Madrid, Ministerio de Educación y Ciencia).

MORENO, A. (2009): La formación del profesorado: una respuesta adecuada a los nuevos tiempos. En M. de PUELLES BENÍTEZ, coord., Profesión y vocación docente. Presente y futuro (Madrid, Biblioteca Nueva - Escuela Julián Besteiro) pp. 43-63.

MOURSHED, M., CHIJIOKE, C. \& BARBER, M. (2010): How the world's most improved school systems keep getting better (McKinsey\&Company) [Consultado el 1 de diciembre de 2010], http://ssomckinsey.darbyfilms.com/reports/schools/How-the-Worlds-Most-Improved-

School-Systems-Keep-Getting-Better_Download-version_Final.pdf

NÓVOA, A. (2009): Para una formación de profesores construida dentro de la profesión. Revista de Educación, 350, pp. 203-218.

OECD (2005): Teachers Matter. Attracting, Developing and Retaining Effective Teachers (Paris, OECD).

OECD (2009): Creating Effective Teaching and Learning Environments. First Results from TALIS (Paris, OECD).

OECD (2011): Building a High-Quality Teaching Profession. Lessons from around the World (Paris, OECD).

PEDRÓ, F. (2006): Un diagnóstico de la situación del profesorado en España desde una perspectiva comparada. Revista de Educación, 340, pp. 243-264.

PEDRÓ, F. y PUIG, I. (1998): Las reformas educativas. Una perspectiva política y comparada (Barcelona, Buenos Aires y México, Paidós).

TIANA, A. (2009): Por qué hicimos la Ley Orgánica de Educación (Las Rozas, Wolters Kluwer). 
TIANA, A. (2010): En búsqueda de la explicación de los resultados educativos: posibilidades y limitaciones de los estudios internacionales de evaluación. Gestión y Análisis de Políticas Públicas, 3, pp. 35-65.

TIANA, A. (2011): Políticas de formación del profesorado y mejora de los sistemas educativos: algunas reflexiones a partir de la experiencia española. Revista Fuentes, 11, pp. 13-27.

VV.AA. (2006): Comentarios a los Informes EURYDICE y OCDE sobre la cuestión docente. Revista de Educación, 340, pp. 19-86.

\section{PROFESIOGRAFÍA}

\section{Alejandro Tiana}

Catedrático de Teoría e Historia de la Educación en la Universidad Nacional de Educación a Distancia (UNED) de España. Ha sido Director del Centro de Altos Estudios Universitarios de la Organización de Estados Iberoamericanos para la Educación, la Ciencia y la Cultura - OEI (20082012), Secretario General de Educación en el Ministerio de Educación y Ciencia de España (20042008), Director del Centro de Investigación y Documentación Educativa (1989-1994), Director del Instituto Nacional de Calidad y Evaluación (1994-1996) y Vicerrector de Evaluación e Innovación de la UNED (1999-2003). Entre 1999 y 2004 fue Chairperson de la International Association for the Evaluation of Educational Achievement (IEA). Es autor o coautor de 19 libros y más de 200 artículos o capítulos de libros sobre diversos temas relativos a la historia de los sistemas educativos contemporáneos, educación comparada o evaluación de los sistemas educativos. Entre sus publicaciones recientes se pueden destacar Por qué hicimos la Ley Orgánica de Educación (2009) y Nuevas miradas historiográficas sobre la educación en la España de los siglos XIX y XX, Madrid (con Guereña, J.L. y Ruiz Berrio, J., eds., 2010). Es director de la Revista Iberoamericana de Educación y miembro del comité editorial de numerosas revistas especializadas nacionales e internacionales, entre ellas la Revista Española de Educación Comparada. Datos de contacto: Departamento de Historia de la Educación y Educación Comparada. Facultad de Educación. Universidad Nacional de Educaci'on a Distancia (UNED). Calle Juan del Rosal, 14. 28040 Madrid. E-mail: atiana@edu.uned.es.

Fecha de recepción: 23 de diciembre de 2012.

Fecha de revisión: 28 de enero de 2013 y 15 de febrero de 2013.

Fecha de aceptación: 22 de febrero de 2013. 